\title{
Analysis and Modelling of the Structural Components of the Elbow Joint
}

\author{
G. Romero, M.L. Martinez, M. Gómez, J.M. Mera \\ CITEF, Railway Technology Research Centre - Universidad Politecnica de Madrid, Spain \\ e-mail: gregorio.romero@upm.es, luisa.mtzmuneta@upm.es, manu.gtrujillo@gmail.com, josemanuel.mera@upm.es
}

\begin{abstract}
A recent application of computer simulation is its use for the human body, which resembles a mechanism that is complemented by torques in the joints that are caused by the action of muscles and tendons. Among others, the application can be used to provide training in surgical procedures or to learn how the body works. Some of the other applications are to make a biped walk upright, to build robots that are designed on the human body or to make prostheses or robot arms to perform specific tasks. One of the uses of simulation is to optimise the movement of the human body by examining which muscles are activated and which should or should not be activated in order to improve a person's movements. This work presents a model of the elbow joint, and by analysing the constraint equations using classic methods we go on to model the bones, muscles and tendons as well as the logic linked to the force developed by them when faced with a specific movement. To do this, we analyse the reference bibliography and the software available to perform the validation.
\end{abstract}

Keywords - Biomedical engineering; Multibody system; Simulation techniques.

\section{INTRODUCTION}

Nowadays, the field of simulation covers fields as diverse as the calculation of mechanisms, the solution to electrical circuits or hydraulic circuits or the heat transfer within an air conditioning installation. This simulation results in some graphs that give us an idea of how a certain system would work in reality, but also helps to optimize its functioning and get some concrete results.

One of the areas of computer simulation is its application to the skeletal-muscular system of the human body [1], which can resemble a mechanism that is complemented with the torques in the joints caused by the action of muscles and tendons, either for surgery training or to understand their behaviour, among others.

The work developed here is aimed at simulating the different parts associated with a joint, such as the elbow, and analysing the mechanism itself as well as the elements that enable it to move. That is, the muscles and tendons and the way they interact with the different parts of the arm.

The main aim is to evaluate the behaviour of a vital part of the human body such as the elbow joint. To do so, the different relationships existing between the bones, muscles and tendons will be analysed as well as the way the human body responds to a thought ('move arm, 'rotate forearm") and transforms it into a movement. To do this, the main aim is to develop the structural-functional components of the human body.

To achieve this aim, the work has been divided into a series of partial objectives. As a starting point, we will first study the anatomy, physiology and mobility of the joints of the forearm as well as the physiological functioning of the muscular activity.

Once the physiology is correctly understood as such, we will analyse the models associated with the muscles, bones and ligaments, either made using simulations or actual prototypes, and then go on to analyse the different simplifications that can be incorporated into the latter. Based on this analysis, we will then be able to obtain the most suitable model for simulating a mechanism like the one presented.

Finally, after analysing the physiology of the elbow and the existing methodologies and mathematical models, we will proceed to develop the simulation of different movements of the elbow in the face of stimulations or external loads - mainly bending-stretching and pronationsupination -, and then go on to compare them with the actual movements of the elbow.

\section{ANATOMY AND Physiology of the Elbow}

When we considered making a dynamic simulation of the elbow after having conducted various studies, we reached the conclusion that the problem needed to be dealt with mathematically as a multibody system. These systems basically comprise various rigid solids that are partly or totally joined to one another by means of kinematic torques. Kinematic torques are perfect joints between solids that allow some degrees of freedom but restrict others. These multibody systems are similar to what might be called articulated bar systems but generalising the concept of articulated bar. Its origin as a specific part of Mechanics can be dated to 1977 during the Congress on the "Dynamics of Multibody Systems" [2].

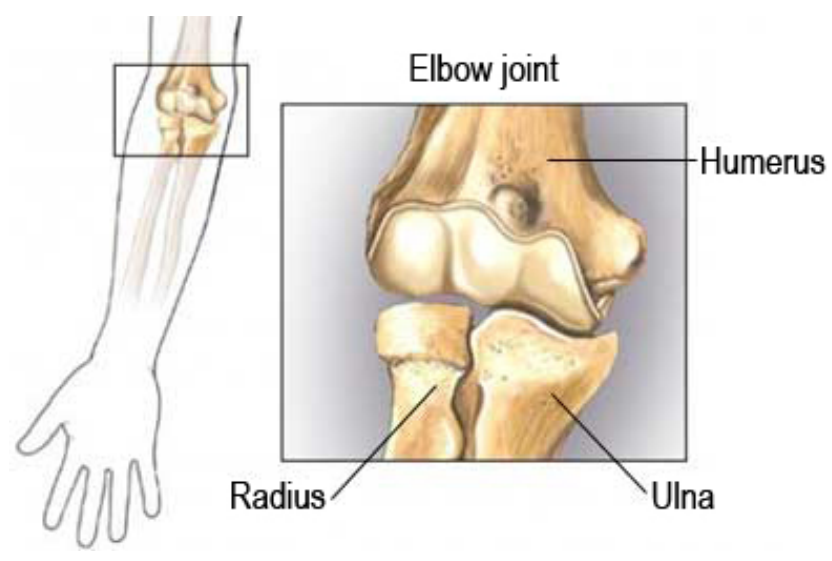

Figure 1. Elbow joints [3] 
Although real joints are based on ligaments, whose function is to keep the joint surfaces in contact, this work will not take them into account, since we will be working with ideal kinematic torques, and will therefore be perfectly defined.

The elbow joint takes the form of a hinge that joins the arm to the forearm and connects the distal part of the humerus to the proximal edges of the ulna and the radius. Three types of joint come together at this point wrapped in a single joint capsule:

\section{A. Humero ulnar joint:}

Two jointed surfaces come into play in this joint, the humeral condyle, part of the humerus, and the sigmoid cavity of the ulna. It is a condyle joint that only allows bendingstretching movement. Strictly speaking, it is the elbow joint. It only has one degree of freedom.
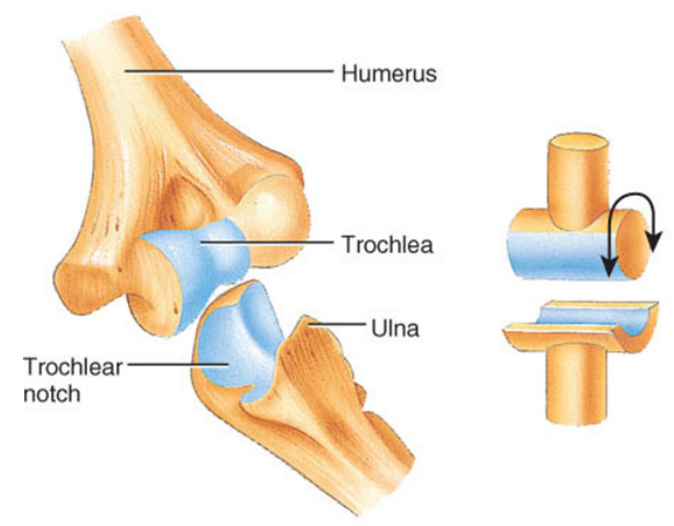

Figure 2. Humero ulnar (Condyle) joint [4]

\section{B. Humeroradial joint:}

Two jointed surfaces also come into play in this joint, which are the radial cupula, on the part of the radius, and the humeral condyle, on the part of the humerus. Its morphology closely resembles a ball or ball and socket-type joint, but it behaves more like a condyle. It has a high degree of mobility.

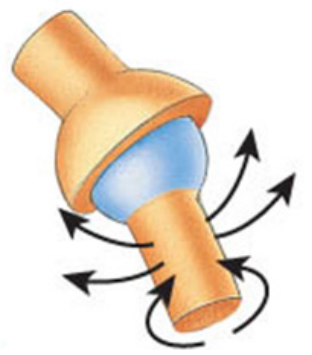

Figure 3. Ball and socket joint [4]

\section{Radio-ulnar joint} distal.

This joint is divided into two joints, the proximal and the

The proximal radio-ulnar joint comprises two jointed surfaces, which are the lesser sigmoid cavity of the ulna, in the form of a hollow cylinder, and the radius head, in the form of a solid cylinder. They, therefore, form a trochoidal joint. This joint is located in the elbow next to the previous two together with the annular ligament. This prevents the radius head from moving downwards and sticking to the ulna, which would prevent sideways movements of the radius on the ulna. It is a trochoidal joint.

The distal radio-ulnar joint (Figure 6) is located at the ends of the ulna and the radius towards the wrist and is also a trochoidal joint. Its jointed surfaces are the ulnar notch of radius and the lateral surface of the radius head. It is also a trochoidal joint.

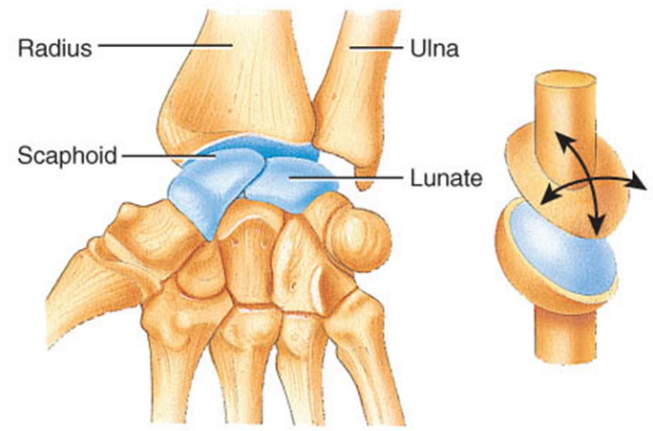

Figure 4. Radio-ulnar joint [4]

\section{ARM-ForEARM MUSCLES}

Although there are over thirty muscles that originate or are integrated in the arm or forearm, this work will only analyse those that are involved in bending, stretching, supination or pronation movements, since it is these actions that will be analysed, the rest being beyond the scope of this work [5].

\section{A. Triceps Brachii}

It starts off in three parts and is the only muscle in the posterior part of the arm. As an example of its movement, it is used to throw objects or push a door to close it. It stems from three points, just below the cavity of the shoulder joint, in the scapula, and from the upper and lower halves of the posterior surface of the humerus. The other end is located in upper posterior area of the ulna, near the point of the elbow.

\section{B. Biceps Brachii}

This muscle is used, for example, to pick something up off the floor or take food to the mouth. It has its origins in both parts of the scapula and is inserted over the medial surfaces of the radius and the ulna.

\section{Brachialis}

It goes under the biceps brachii and is the main flexor of the elbow joint, it being one of the muscles used to take food to the mouth.

It originates in the anterior surface of the lower part of the humerus and its end in the area before the upper part of the ulna. 


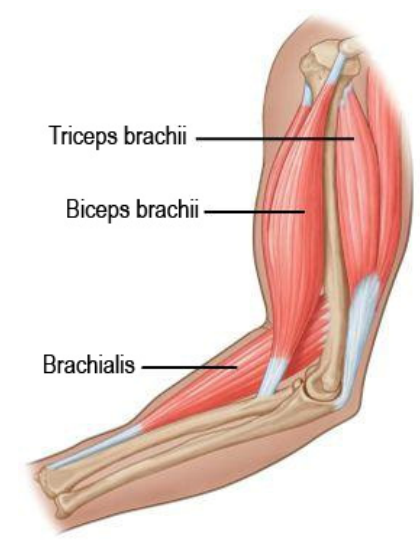

Figure 5. Tríceps, Biceps and Brachialis muscles [6]

\section{Anconeus}

This muscle, which most people have, but not everybody, is agonist of the brachial triceps brachii. It stems from the inferolateral end of the humerus (epicondyle) and is integrated into the posterior surface of the ulna, near the olecranon.

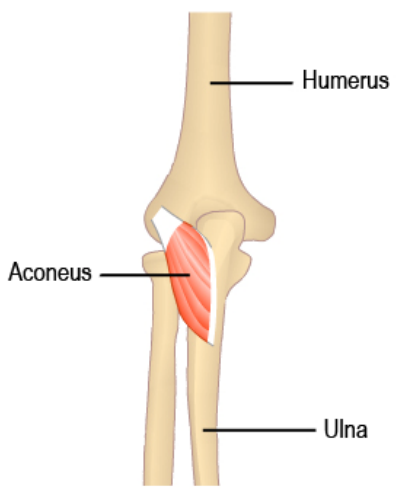

Figure 6. Anconeus [7]

\section{E. Brachioradialis and Short Supinator}

The main action of the long supinator, also called the brachioradialis muscle, is bending, as for example, turning a doorknob or a screwdriver. It becomes a supinator when there is maximum pronation and a pronator when there is maximum supination. It stems from the lower lateral part of the humerus a few centimetres above the elbow and is integrated into inferolateral end of the radius.

The short supinator (Brachioradialis) is an agonist muscle of the short supinator and stems from the inferolateral end of the humerus (epicondyle) and the posterior surface of the olecranon, while its other end is located on the dorsal and lateral surface of the upper third of the radius.

\section{F. Pronator Teres and Pronator Quadratus}

The pronator teres is the main actuator in the pronation movement, as, for example, when pouring liquid into a container. It stems from the inferomedial end of the humerus (medial epicodyle) and its other end stems from the middle part of the lateral surface of the radius.
The pronator quadratus is the secondary pronator of the forearm and is fairly distant from the elbow, which means a slight contraction produces pronation. It stems from the anterior surface of the lower quarter of the ulna and its other end stems from the distal quarter of the anterior surface of the radius.

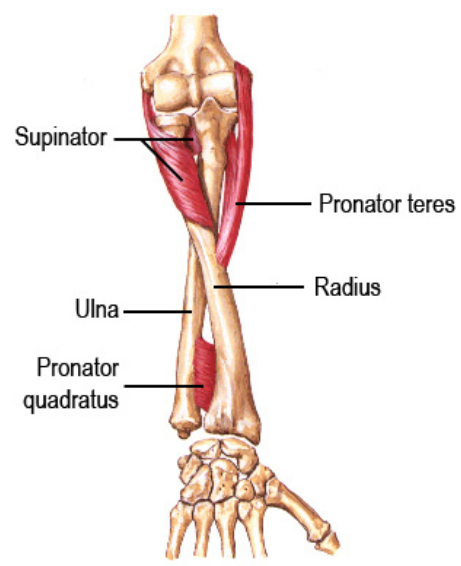

Figure 7. Supinator and Pronators muscles [8]

\section{Muscle Model. The HILL-Type Model}

For a muscle to be able to function and interact properly, a nervous impulse is required. This is generated in the form of an action potential by the nerves and neurones until it reaches the motor plate or neuromuscular joint. Depending on the number of fibres activated, the muscular tension will be higher or lower. So, it can be said that the nerve stimuli are added together and so it can be assumed that the level of mechanical tension is a response to a certain nervous impulse in the form of a voltage.

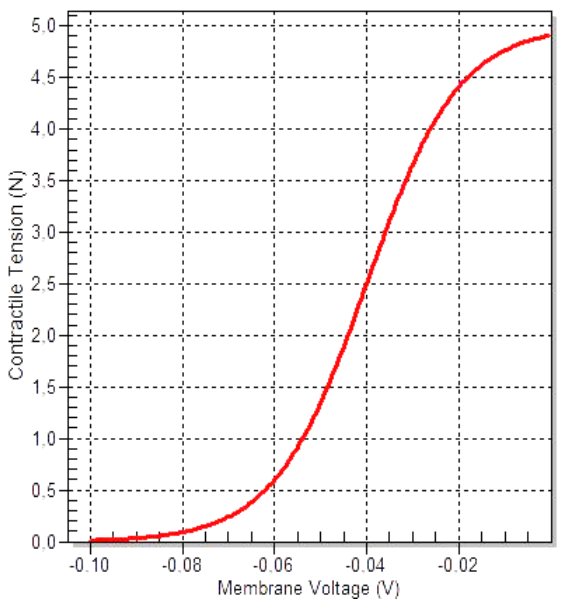

Figure 8. Stimulus-Tension Curve

The voltage corresponds to the potential difference in the muscular membrane between the neural button and the fibre wall. The beginning results in a negative value, in principle, to represent the voltage at rest. The greater the voltage, that is, the greater the stimulus, the greater the tension because there are more fibres activated. The curve is asymptotic towards a maximum value of tension corresponding to the maximum force that the muscle can develop. 
As for muscular models, a variety of mechanical models of muscle have evolved to describe and predict tension, based on some input stimulation. Crowe [9] and Gottlieb and Agarwal [10] proposed a contractile component in conjunction with a linear series and parallel elastic component plus linear viscous damper. Glantz proposed nonlinear elastic components plus a linear viscous component. Winter [11] has used a mass and a linear spring and damper system to simulate the second-order critically damped twitch. In 1949 Hill [12] proposed such a lumpedelement model for the muscle. The Hill model is still in use today, and it remains the most popular form of lumpedelement model for the muscle.

There are actually two canonical forms of Hill's model, but these forms are mathematically equivalent under a suitable change of variables, McMahon [13]. The canonical form used here is the easier one to apply to a muscle when it is regarded as composed of multiple motor units acting in parallel with one another.

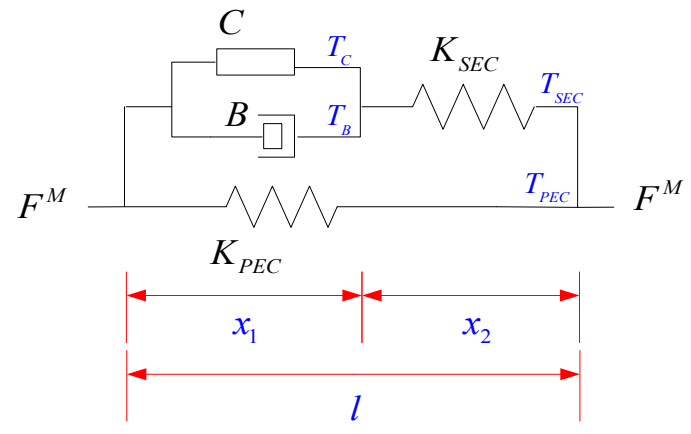

Figure 9. Hill's model

Figure 1 illustrates the Hill model. There are four basic elements in it: the contractile element ' $\mathrm{C}$ ', the damping element ' $\mathrm{B}$ ', the series elastic component ' $\mathrm{K}_{\mathrm{SEC}}$ ', and the parallel elastic component ' $\mathrm{K}_{\mathrm{PEC}}$ '.

\section{A. Contractile element}

The contractile element ' $\mathrm{C}$ ' is the "active" element in an extrafusal motor unit and it corresponds to the role played by voltage in an electronic circuit, responding to motor neuron inputs by contracting. Thus, the tension ' $\mathrm{T}_{\mathrm{C}}$ ' that it produces always acts to try to shorten the muscle. Finally, this element is unable to produce an extension force.

\section{B. The damper element}

It is an empirical factor that muscle tension during contraction and the speed of the contraction are coupled to each other. Hill found that the relation between them follows a characteristic hyperbolic equation, now known as Hill's equation. Such elastic elements, like the damper coefficient ' $\mathrm{B}$ ' is a function of the contraction speed, therefore it is a nonlinear damper.

\section{The elastic elements}

A muscle when passively stretched exhibits an elastic restoring force that tends to return the muscle to its original length. In part this force is due to stretching the connective tissue that surrounds the muscle fibres. In part it may be due to stretching the tendons which terminate muscle tissue and attach it to the bone. There is a reason to believe that the muscle fibres themselves are at least partly elastic. It is this elastic restoring force that is represented by the elastic elements (springs) in the Hill model. It is not completely correct to assign these elements to any particular physical source, but we may regard the ' $\mathrm{K}_{\mathrm{PEC}}$ ' as being mostly due to the connective tissues and the ' $\mathrm{K}_{\mathrm{SEC}}$ ' as being primarily dominated by tendon fibres terminating specific motor units. We should note that ' $\mathrm{K}_{\mathrm{PEC}}$ ' and ' $\mathrm{K}_{\mathrm{SEC}}$ ' are functions of lengths and therefore are non-linear springs.

The net force obtained by the muscle is a combination of that obtained in the parallel element. In short, this is a good representation of the active and passive tensions, which are represented in the following figure [14].

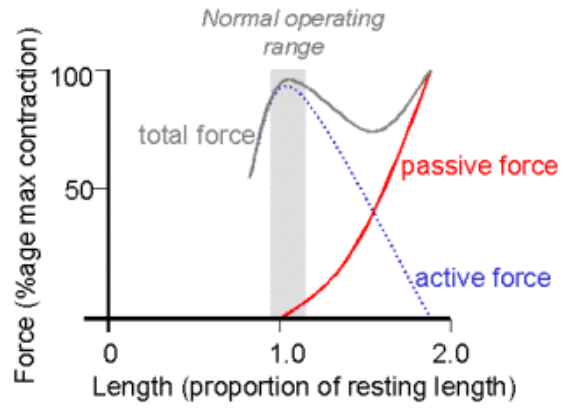

Figure 10. Active and passive tension in the muscle

The Hill model is widely accepted as the best physicalmathematical representation of muscle behaviour. It should be pointed out that this model is purely quantitative; Hill did not propose any values for the spring constants or for any other parameter.

As a complement to his model, this author proposed a state equation applicable to the skeletal muscle that has been stimulated to reach tetanic contraction, which relates the tension reached in the muscle with the speed of contraction:

$$
(v+b)(F+a)=b\left(F_{0}+a\right)
$$

, where ' $F$ ' is the tension or load in the muscle, ' $v$ ' is the speed of contraction, $F_{0}{ }^{\prime}$ is the maximum isometric tension the muscle can reach and ' $a$ ' and ' $b$ ' are parameters to be fitted. The graphic representation of this equation is shown in Figure 11.

The main defect of the Hill model is that it does not include an element to dissipate energy, which means that effects like the warming of muscles after exercise cannot be explained. In spite of this, as already stated, it is the most widely accepted model. In addition to the information of the Hill model, Wells [15] writes a complete set of equations describing each element. 


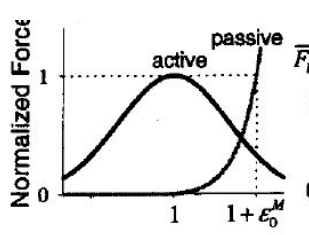

Normalized Length
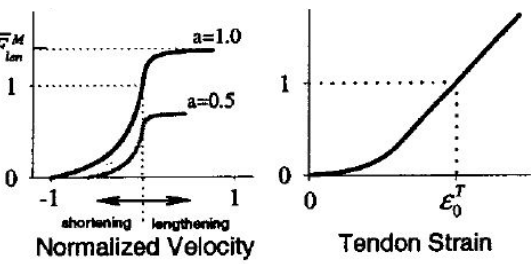

Figure 11. Representation of the Hill equation [16]. a). Muscle F-L, b). Muscle F-V, c). Tendon

\section{FULL FOREARM AND ELBOW MODEL}

When each of the structural elements (bones) and functional elements (muscles) have been analysed the modelling can begin.

As already described in the chapter on anatomy, the forearm has four joints: humeri ulnaris, humeri radialis, lower humerri ulnaris and the upper humeri ulnaris, which is why a two-bar model has been proposed to simulate this mechanism.

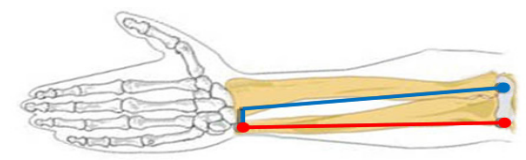

Figure 12. Trapezoidal model of the forearm

This model is made up of twelve variables to define it in space, and three ball joints, which adds nine constraint equations. If, in addition, we impose the condition that the angle of the red bar (ulna) is constant (since it is prevented from rotating around itself), a new constraint equation is created. In all, there are ten equations and twelve variables making a two-degree of freedom system, degrees which correspond to the bending-stretching and pronationsupination movements.

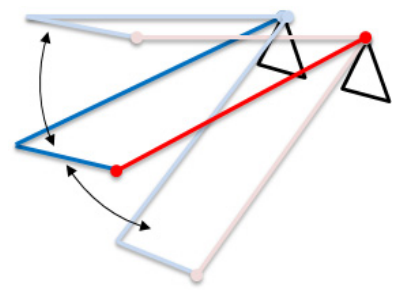

Figure 13. Degree of bending-stretching freedom

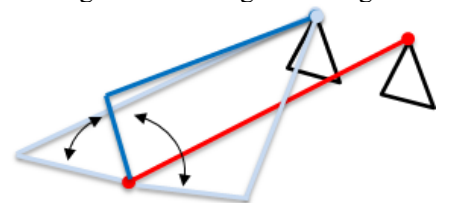

Figure 14. Pronation-supination degree of freedom

In order to build a correct simulation model the reference system chosen for the mechanism is that shown in the following figure:

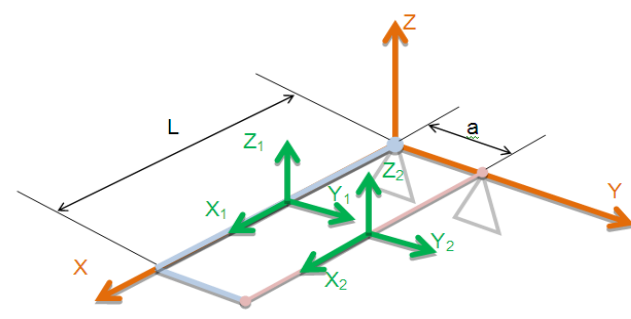

Figure 15. Reference systems of the mechanism

The constraint equations used are as follows and describe each of the joints associated with the physiology of the elbow.

It is first essential to define the matrix ' $R_{\alpha, \beta, \gamma}$ ', which represents the turning matrix of the angles ' $\alpha$ ', ' $\beta$ ' and ' $\gamma$ ' that will be used to define the different equations.

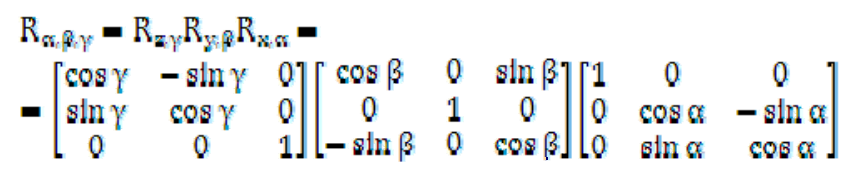

(2)

Regarding the ball joint in the radius (blue bar), it is essential to define its position in respect of the reference system of the mechanism.

$$
\left[\begin{array}{l}
\emptyset \\
0 \\
\emptyset
\end{array}\right]=\left[\begin{array}{l}
x_{1} \\
y_{1} \\
z_{1}
\end{array}\right]+R_{\kappa 1 / 1 / \gamma^{1}} \cdot\left[\begin{array}{c}
-L / 2 \\
0 \\
0
\end{array}\right]
$$

Likewise, the ball joint in the humeri radialis (red bar) is that shown below, with the difference that this joint has to consider the distance between both joints of the ulna and the radius:

$$
\left[\begin{array}{l}
0 \\
0 \\
0
\end{array}\right]=\left[\begin{array}{l}
\mathrm{x}_{2} \\
\mathrm{y}_{2} \\
z_{2}
\end{array}\right]+R_{\kappa 2, \beta 2, \gamma 2} \cdot\left[\begin{array}{c}
-\mathrm{L} / 2 \\
0 \\
0
\end{array}\right]-\left[\begin{array}{l}
\mathrm{a} \\
0 \\
0
\end{array}\right]
$$

At the other end of both bones, the equation corresponding to the ball joint that is common to both can be easily found by just imposing the condition of equality of positions on both points:

$$
\left[\begin{array}{l}
0 \\
0 \\
0
\end{array}\right]=\left[\begin{array}{l}
\mathrm{x}_{1} \\
\mathrm{y}_{1} \\
\mathrm{z}_{1}
\end{array}\right]+\mathrm{R}_{\kappa 1, \beta 1 \gamma 1} \cdot\left[\begin{array}{c}
\mathrm{L} / 2 \\
\mathrm{a} \\
0
\end{array}\right]-\left(\left[\begin{array}{l}
\mathrm{x}_{2} \\
\mathrm{y}_{2} \\
\mathrm{z}_{2}
\end{array}\right]+\mathrm{R}_{\kappa 2, \beta 2, \gamma 2} \cdot\left[\begin{array}{c}
\mathrm{L} / 2 \\
0 \\
0
\end{array}\right]\right)
$$

Finally, and in accordance with the physiognomy shown, a constraint must be imposed on the rotation of the ulna around itself (red bar). This means that an imposition must be applied to the vector ' $k$ ' so that it will always be coplanar with the vectors ' $i$ ' and ' $k$ '. 


$$
\left|\begin{array}{cccc}
1 & 0 & 0 \\
0 & 0 & & 1 \\
& \mathrm{~K}_{\kappa z, \beta z, \gamma z 2} & {\left[\begin{array}{c}
0 \\
0 \\
1
\end{array}\right]} &
\end{array}\right|=0
$$

To properly validate the model various basic movements have been simulated in accordance with the model described, bearing in mind that the principal movements of the forearm over the arm are bending (as opposed to stretching) and pronation (as opposed to supination). Animatlab software has been used to view the model as it lets the different defined elements be introduced.

\section{RESULTS}

The lack of numerical parameters in the Hill model to create a realistic model was a major problem when it came to fitting it. However, we were able to obtain some values experimentally to be implemented in the Hill model, which provided good results in simple situations of movement $\left(K_{s e}=1000 \mathrm{~N} / \mathrm{m}, K_{p e}=200 \mathrm{~N} / \mathrm{m}\right.$ y $\left.B=120 \mathrm{~N} \cdot \mathrm{s} / \mathrm{m}\right)$.

\section{A. Impulse over the biceps brachii}

If a bending movement is to be simulated, in principle it is the biceps muscle that has to act with the possible help of the brachialis. To do this, a stimulus is created in the form of a constant current over the neurone controlling the muscle. In this case, two constant impulses are applied to two neurones, since the biceps, in spite of being a single muscle, has been simulated as two independent actuators due to the two insertions it has in the ulna and radius bones. The intensity of both impulses is the same, with a value of $27 \mathrm{nA}$.

Using this impulse the simulation gives a fairly realistic result. The arm is shown in an ascending movement rotating around the axis of the hinge that represents the humeri ulnaris joint, and over the ball joint that simulates the humeri radialis joint. The following two images show the initial and final positions of the model in this simulation.
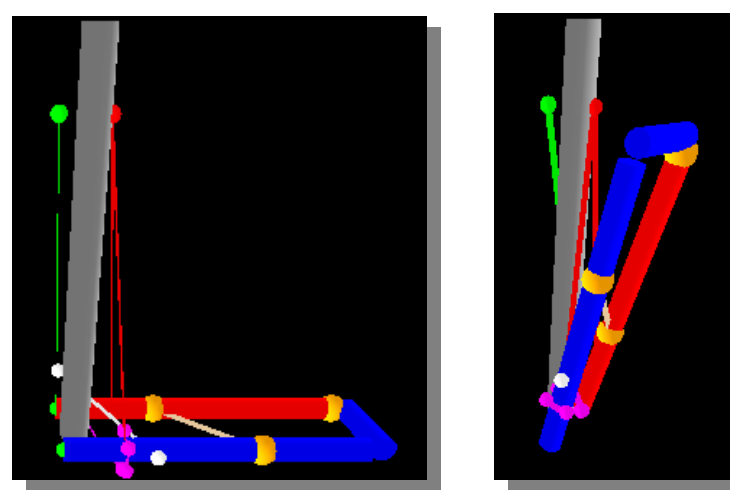

Figure 16. Position (a). before, b). after) the impulse over the biceps

The results in graphic form coincide logically with the simulation:

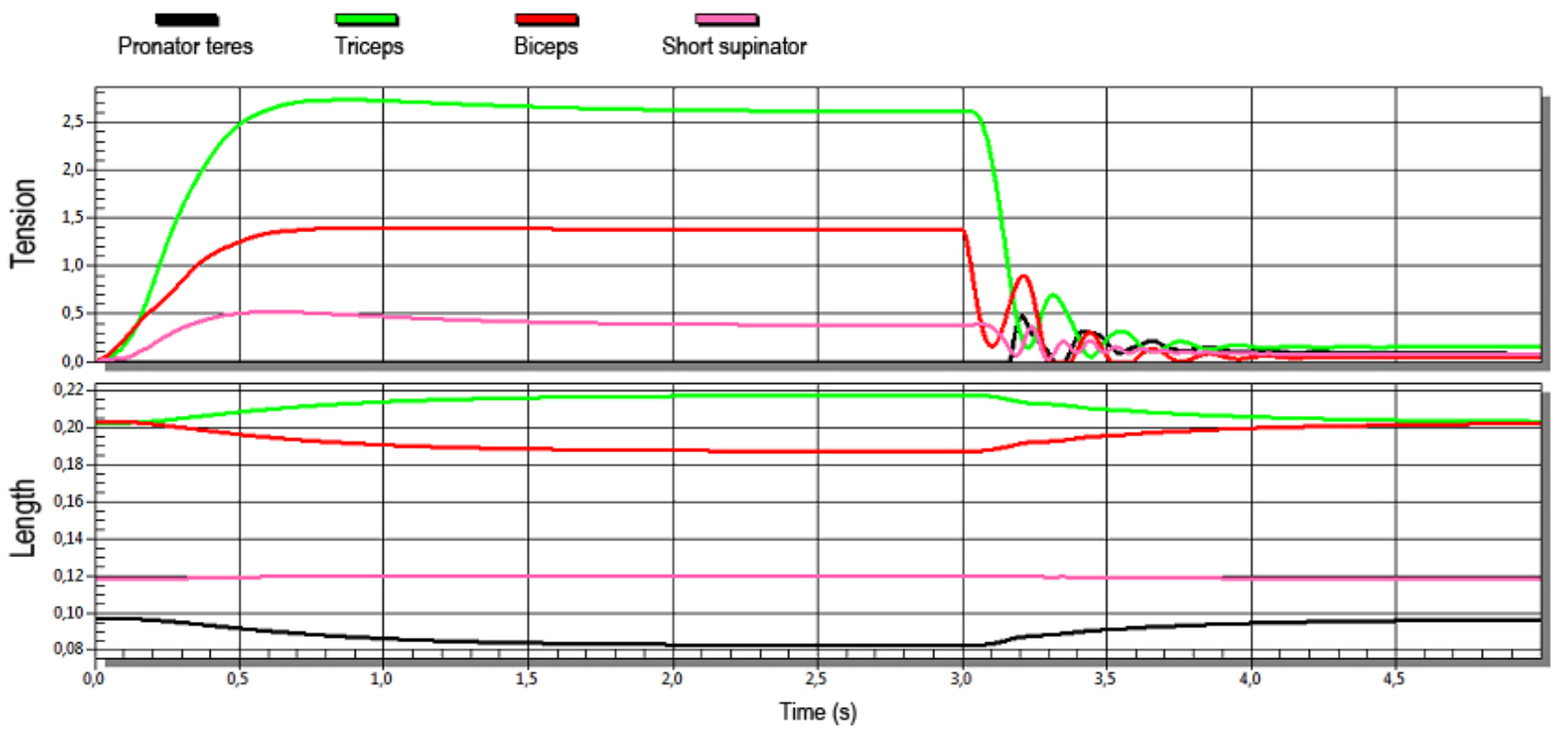

Figure 17. Tensions and muscle lengths in response to the impulse over the biceps

It can be observed from the graph that the biceps becomes approximately two centimetres shorter while the triceps becomes longer to approximately the same extent. On the other hand, the tension developed in the biceps is a growing tension although slightly oscillating, the same as the triceps and the pronator teres. It is important to point out that although the biceps appears with a lower tension than the triceps, the former is represented y two equal muscles that produce tension. This means that the real contribution is double to what appears in the graph and so the result is greater than that obtained for the triceps.

When the impulse stops, after five seconds the elastic elements of the muscle models go back to their position of equilibrium, which takes the mechanism, the arm, to its 
initial position in a bending of $90^{\circ}$. As can be seen, in the graphs the muscles go back to zero tension and their normal length.

The angle formed by the forearm in respect of the humerus also shows the expected behaviour: it increases over time but is non-oscillating and reaches a state of equilibrium at around $50^{\circ}$.

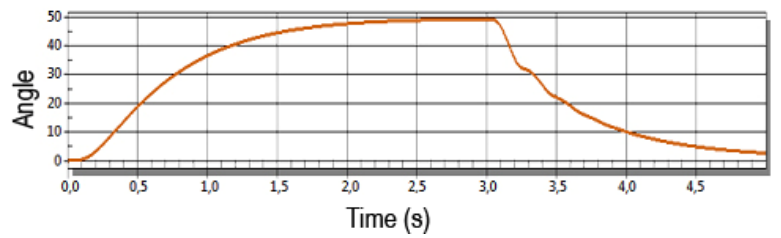

Figure 18. Angle between forearm and the horizontal with an impulse over the biceps

\section{B. Pronation with free supination}

Pronation is an essential movement in a human being's life. However, although bending and stretching are always achieved with the same group of muscles, pronation and supination depend on whether the forearm is under bending or stretching. In order to be able to analyse the pronation and pure supination this movement must be analysed at a bending angle of $90^{\circ}$, to ensure that there is no contribution from the shoulder muscles in this movement.
For this reason, it was decided to block the hinge that represents the humeri ulnaris joint in the ulna.

In this section, this action is simulated with a $32 \mathrm{nA}$ nerve impulse over the pronator teres that stops after five seconds. The initial and final positions are as follows:

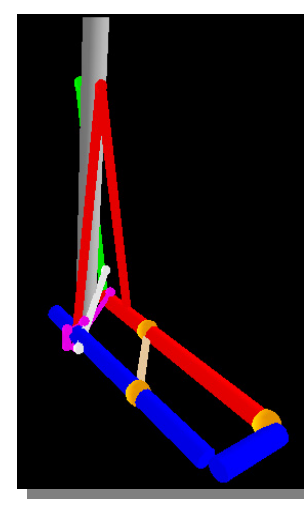

a).

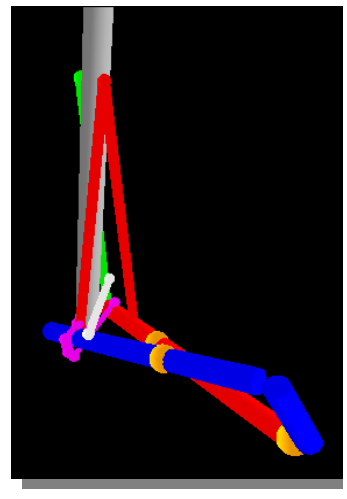

b).
Figure 19. Position (a). initial, b). final) with pronation and free supination

If we again add the results for tension and length in graphic form (Figure 20), more conclusions can be reached:

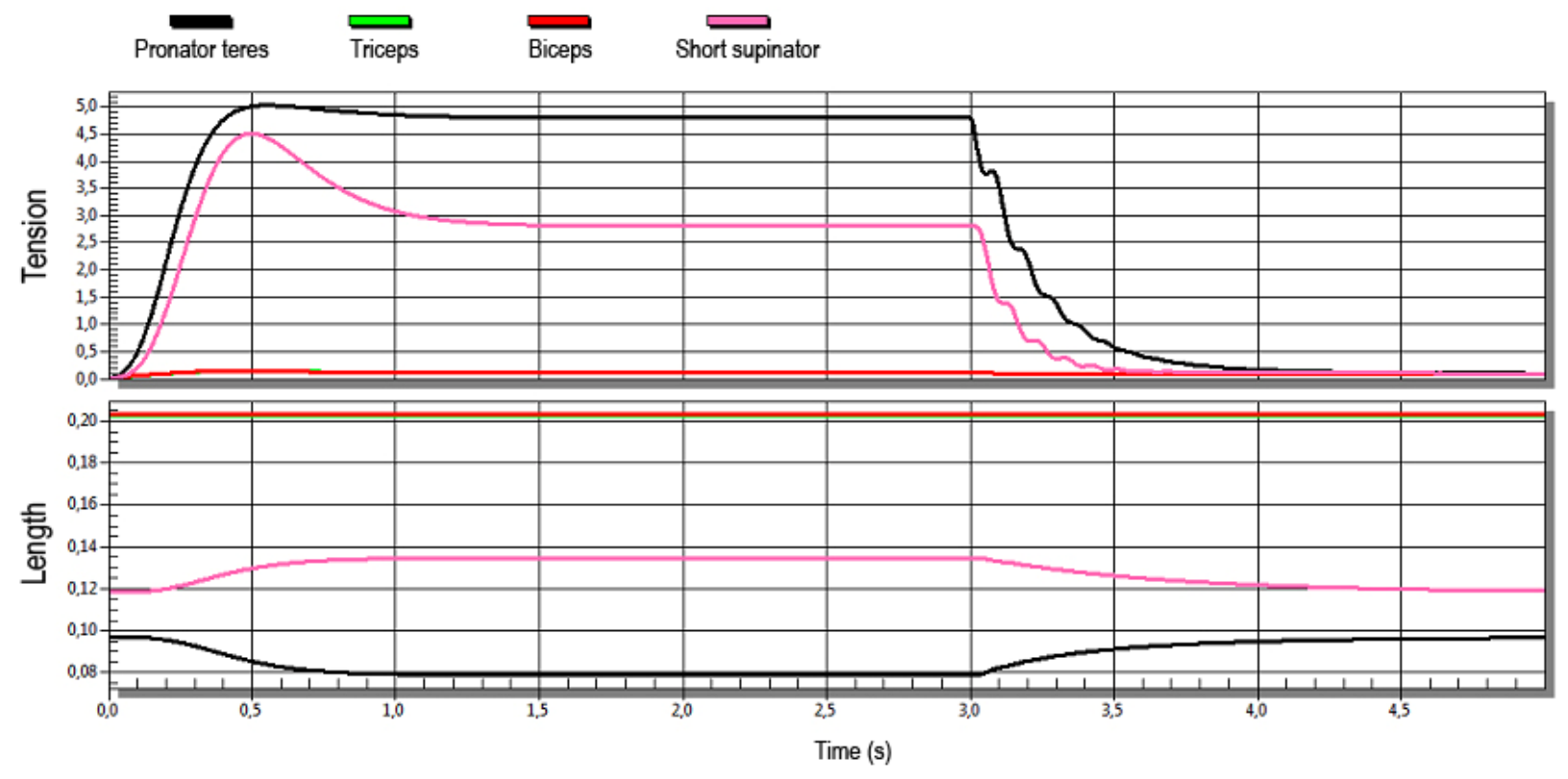

Figure 20. Muscle tension and length with pronation and free supination

From the above figure it can be seen that the greatest contribution in tension is due to the pronator teres. The long supinator also has a certain tension value due to the fact that it can be seen to be stretched and adopts a passive tension. The triceps brachii and biceps muscles scarcely make any contribution nor can they be seen to be affected in the simulation since their length does not vary during the entire movement.
The return to equilibrium is again due to the passive tension that each muscle has when the nerve impulse over the pronator teres stops. The position of equilibrium in the absence of external forces coincides with the initial position and it can be seen that all the muscles, in particular the pronator and the supinator, return to zero tension and the nominal length in just over one second. 


\section{CONCLUSIONS AND FUTURE WORKS}

If we examine the different analyses and studies carried out during this work, as well as the different simulations performed on the elbow model presented, a series of conclusions can be reached from the work carried out, some of which are set out below.

Firstly, the models available for representing the muscles and the nervous system were analysed, checking that there were qualitative muscle and neurone models that responded to experimental behaviour, even though these models were not precisely parameterised.

To this end, the effects of these muscles were simulated independently as well as the behaviour of the arm when faced with a vertical external force. As an application of these effects, we simulated a combined movement of bending with pronation.

After performing the initial analysis in the project, one of the things observed was the fact that all the muscles, whether active or not, develops tension in the face of any change in the position of the forearm compared to when it is at rest.

On the other hand, from an engineering point of view and as a link to the field of medicine, it has been observed that the force developed by the active muscles depends on the intensity of the nerve impulse and is highly sensitive to small variations of this impulse. In this respect, it has also been observed that how the action of the triceps, as it is a muscle with a single insertion, causes pronation due to the passive tension of the pronator muscles and the interosseous membrane.

Although at the beginning it was thought that the model would not give rise to many problems (articulation between two elements), it has been seen that although elementary movements such as pronation and supination have a main actuator muscle, each muscle does not have a single function, but that practically every muscle intervenes in any movement, either actively or passively.

One of the future lines of research deemed to be most significant and which we have already mentioned in the conclusions, consists in parameterising more correctly the muscles and the neurones, since they have been closely studied from a medical point of view but very little from an engineering one. In addition, if a fuller model of the arm is to be developed, all the remaining muscles in the forearm would need to be included. Moreover, to build the proposed model accurately, it is essential to improve the parameterisation and the feedback elements existing in the nerve control to control the closed loop.

\section{REFERENCES}

[1] Mendoza-Vazquez, J.R., Escudero-Uribe, A.Z., Tlelo-Cuautle, E. (2008). Modeling and Simulation of a Parallel Mechanical Elbow with 3 DOF. Electronics, Robotics and Automotive Mechanics Conference, pp.455-460.

[2] International Symposium on Dynamics of Multibody Systems. Munich (Germany). 1977.

[3] Nucleus Medical Media, Inc.

[4] Larson K. (2012). Fibrous Joints. StudyBlue website. <http:// http://www.studyblue.com/>. Last accessed $31^{\text {st }}$ Dec. 2013.

[5] Free virtual human anatomy website. <http://www.innerbody.com/>. Last accessed 31 $1^{\text {st }}$ Dec. 2013.

[6] Arnott K. (2012). Muscles. StudyBlue website. <http:// http://www.studyblue.com/>. Last accessed 31 $1^{\text {st }}$ Dec. 2013.

[7] GetBodySmart website - An Online Human Anatomy and Physiology Texbook. <http://www.getbodysmart.com/>. Last accessed $31^{\text {st }}$ Dec. 2013.

[8] Human Anatomy website. <http://www.knowyourbody.net/>

[9] Crowe, A. (1970) A Mechanical Model of Muscle and its Application to the Intrafusal Fibres of Mammalian Muscle Spindle, J Biomech 3:583,592.

[10] Gottlieb, G. L. and G. C. Agarwal (1971) Dynamic Relationship between Isometric muscle Tension and the Electromyogram in Man, J Appl Physiol 30:343-351.

[11] Winter, D. A. (1976) Biomechanical Model Related EMG to Changing Isometric Tension, in Dig. $11^{\text {th }}$ Int. Conf. Med. Biol. Eng. pp. 362-363.

[12] Hill A. V. (1949) The abrupt transition from rest to activity in muscle, Proc. Roy. Soc. London B, vol. 136, issue 884, pp. 399-420

[13] McMahon T.A.(1984) Muscles, Reflexes, and Locomotion, Princeton, NJ: Princeton University Press, pp. 23-25

[14] Brown University. http://www.brown.edu/. Last accessed 31 $1^{\text {st }}$ Dec. 2013.

[15] R. Wells, (2003) Kinetics and Muscle Modeling of a Single Degree of Freedom Joint, Part I: Mechanics. <http://www2.cs.uidaho.edu/ >. Last accessed 31 ${ }^{\text {st }}$ Dec. 2013.

[16] Thelen, D.G. (2003). Adjustment of Muscle Mechanics Model Parameters to Simulate Dynamic Contractions in Older Adults. J Biomech Eng 125(1), 70-77. 